\title{
Simulation Modeling Methods and Tools in the Study of Electronics Preproduction
}

\author{
Alexey E. Kurnosenko ${ }^{1 *}$, Ivan V. Levin ${ }^{1}$, Valeria $O$. Semenyakina ${ }^{1}$, Evgeny $_{\text {R. Zakharov }}{ }^{1}$, \\ and Artem E. Sherstyuk ${ }^{1}$ \\ ${ }^{1}$ Bauman Moscow State Technical University, 2nd Baumanskaya str., 5/1, 105005, Moscow, Russia
}

\begin{abstract}
The paper is devoted to the selection and application of simulation methods and tools when creating a digital model of the production floor and the preproduction of the assembly process of electronic modules on printed circuit boards. The advantage of simulation modeling for creating a digital manufacturing model is shown. The introduction of simulation methods in the educational process is considered. Using the developed digital model for the manufacturing of a typical product as an example, the functional capabilities of the Plant Simulation module from the Tecnomatix software package developed by Siemens PLM Software are considered. The main simulation results in terms of productivity of the designed production floor are considered. The digital model was improved in order to increase the technological equipment utilization rate and the total productivity of the assembly floor.
\end{abstract}

\section{Introduction}

The modern educational process has a pronounced tendency to introduce various modeling tools for studying complex technical objects. In particular, this applies to the teaching of technical disciplines related to complex advanced technologies, production methods and structures. As a rule, when describing such objects, analytical models are either completely absent or only partially can reflect the complex relationships of production objects, as well as determine the response of the production structure to disturbing influences. The study of a really working production also faces unavoidable problems associated primarily with the fact that the process parameters for an outside observer in most cases are not formalized and clearly traceable, which makes it difficult to understand the observed production processes. In addition, it is impossible to conduct learning experiments in a running production facility in order to identify a response to disturbing influences. It is also important to consider the economic and organizational difficulties of such a learning option.

A solution to this problem is possible when complex technical objects simulation methods and tools are introduced into the educational process. Provided that the model is adequate to the real production process, it is able to act as a substitute to real running production for educational purposes. The digital model will allow not only to comprehensively study the processes occurring in the production, but also to clearly

* Corresponding author: akurn@ bmstu.ru 
formalize the input and output parameters of the process, as well as to set up a series of "what if" experiments, applying various disturbing influences to the model input. All this in a complex will give the student a complete idea of the subject being studied. This paper discusses the implementation of this concept on the example of building a digital model of discrete production of electronic modules.

It is important to note that a similar tendency of "digitalization" is also observed in the production structures themselves, which is aimed at accelerating the new products introduction to the market and reducing costs. Digitalization, carried out within the concept Product Lifecycle Management (PLM), covers, in particular, such important stages as preproduction, production planning and the actual production process itself. At that, the modern "Industry 4.0" concept of organizing efficient production provides for a virtual representation in the form of a digital model of not only the product itself, but also its entire environment, including objects involved in the production process - starting with equipment, fixtures and tools, storage area, material flows, personnel and up to the entire production floor as a whole.

In this context, by preproduction and production planning we mean a systematic activity that allows to calculate and predict the goals, stages, composition and characteristics of the production process and floor under such changes as a new product introduction, introducing new equipment, fixtures and tools, eliminating bottlenecks in the existing production system etc. [1].

The practice of modern production often demonstrates the optionality and intuitiveness of measures for the development, planning and modernization of production processes. This activity takes place in conditions of limited digitalization, which often stops at the stage of creating a product design model, which prevents the high competitiveness of enterprises or, in any case, does not allow to fully achieve it. Using a systematic approach to the preproduction and production planning, fully digitalizing, including the production component, and allocating the appropriate resources for this task before the physical implementation of changes, it is possible to avoid costly corrective actions in the production process and thus reduce the time and costs for manufacturing the product and speed up profit-making [2].

The implementation of these approaches, consistent with the modern "Industry 4.0" concept, should be accompanied by the training of appropriate engineering personnel competent not only in the field of electronics manufacturing technology, but also in the field of modeling, analysis, and optimization of technical objects. Thus, in addition to product and manufacturing, digitalization should also relate to relevant educational technologies [2-5].

Digital preproduction and planning software and IT infrastructure provided by "Digital Manufacturing" form the main building block for organizing effective "digital" collaboration between design and preproduction / production planning stages. Providing preproduction and production planning specialists with access to digital data on products directly in the planning environment allows them to more easily and thoroughly check the data for compliance with production requirements. Using three-dimensional digital models, planners can develop their requirements and proposed modifications - especially in terms of design for manufacturability - at a much earlier stage and on a more reliable basis than before [6].

Considering the above, in this paper we will focus on digital modeling methods and tools for the electronics manufacturing process and preproduction using the example of electronic modules on printed circuit boards. The methods and tools under consideration, in addition to the full reflection of all aspects of digital manufacturing, should allow its study, analysis and modernization, as well as be suitable for use in engineering education when 
training specialists in production organization, as well as production engineers for automated manufacturing.

\section{Formulation of the problem}

The problem in this study was formulated as follows: it is required to create a digital model of the assembly area of electronic modules on printed circuit boards using mixed technology (with surface mount and through hole technologies), which reflects the totality of production objects - equipment, fixtures and tools, storage area, components, work in progress and finished products, personnel - as well as temporal and logistic relationships between them. The model should adequately describe the preproduction, the assembly process and be capable of issuing main time parameters for assembly production - cycle times for each operation and the total assembly performance, understood as the number of manufactured products per unit time.

\section{Choice of modeling method}

To solve the problems of modeling objects, it is fundamentally possible to use the methods of analytical and simulation modeling. The first group relates to static methods, where the resulting model parameters are calculated using formulas with a strictly determined result. Using such methods, it is impossible to consider complex dynamic processes subject to external and internal perturbations. It is these processes that take place in the electronics manufacturing, which are characterized by technological process variability, frequent changeovers, forced downtime due to equipment failures, the impact of component quality, the human factor in operator work, etc. Most of the factors listed above are probabilistic and have variability over time. Consequently, the development of a model adequate to real production will be possible using more flexible methods of complex (simulation) modeling, which allow to build a model based on a sequence of events, control it over time, introduce random fluctuations and impose resource and other restrictions. The results of simulation modeling, in contrast to analytical one, are the product of statistical processing of data observed and recorded during the work of the modeling program.

In any study related to the use of simulation modeling, the following stages are established:

1. Model development and parameterization.

2. Implementation of a model using a software modeling system.

3. Model analysis.

4. Carrying out experiments on a running model.

5. Results analysis.

\section{Choice of modeling tools}

On the market there are a number of computer-aided design and preproduction systems. When choosing a specific computer-aided system, preference was given to systems for comprehensive support of the product life cycle with the most seamless transition from a set of design models to a production simulation model. Based on this criterion, Siemens PLM Software products were selected for the project: computer-aided design software Solid Edge for developing models of product, equipment, fixtures and tools, as well as the Plant Simulation module [7,8], which is part of the Tecnomatix integrated modeling and optimization system for digital manufacturing - to create a simulation model of the assembly area. Both software products can run under Teamcenter PLM system, providing 
even closer integration of various models. As to solving the formulated problem, the Plant Simulation module allows:

- to create structured, hierarchical models of production objects, manufacturing lines and processes;

- to conduct simulation using built-in tools;

- to carry out experiments and work out scenarios of the "what if" type without interfering with the operation of already running production systems;

- to use an extensive set of analytical tools (bottleneck analysis, statistics and graphs, including equipment utilization rate) to evaluate various production scenarios;

- to present models in 2D and 3D.

The work was implemented in the framework of the final qualification work of the master Levin I.V. Student software versions were used.

\section{Modeling}

In order to implement the stages of simulation modeling described in Section 3 using the above-mentioned software, the following sequence of basic actions was taken:

1. Manufacturing process description

2. Choice of technological equipment and equipment

3. Creation of 3D-models for products, equipment, fixtures and tools

4. Seeding the Plant Simulation database

-Creating moving objects

- Creating material flow objects

- Setting parameters of operation, changeover, failures

- Kinematic setup

-Creating event handlers

5. Manufacturing line setup

6. Personnel setup

7. Start simulation

8. Results analysis

9. Conclusions making

Next will be a brief discussion of the key stages of the work done.

\subsection{Manufacturing process description}

The selected automated assembly process included the following main operations: screen printing of solder paste, placement of surface-mounted components, reflow soldering, manual insertion / soldering of through hole components, cleaning and control.

\subsection{Choice of technological equipment}

The following models were selected as technological equipment for performing the main operations of the manufacturing process:

- component pick-and-place machine Philips Topaz;

- solder paste screen printing machine Ekra X5 Professional;

- reflow oven Ersa HotFlow 3/14e. 


\subsection{Creation of 3D-models for products, equipment, fixtures and tools}

Typical product or the assembly object is an electronic module on a printed circuit board that is part of the room temperature control system. This module is characterized by onesided mounting of mixed components (there are surface- and through hole mounted components). Fig. 1 shows a general view of a 3D model of an assembly created in Solid Edge CAD.

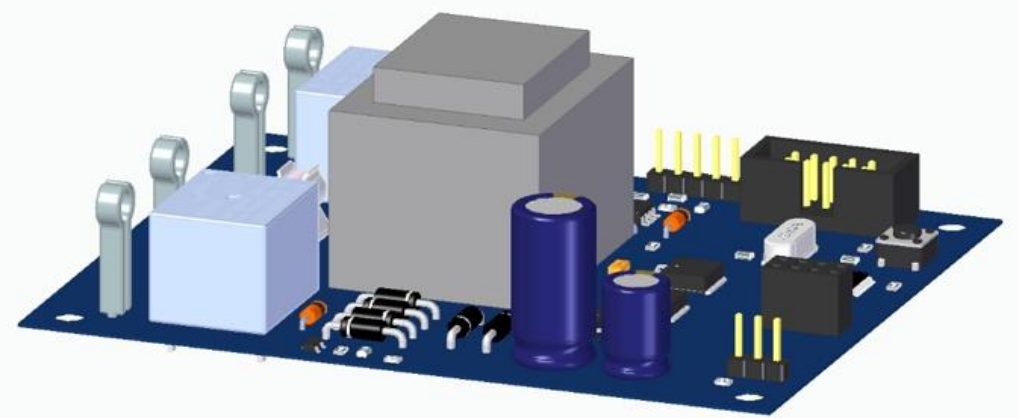

Fig. 1. 3D-model of the production object - an electronic module of the room temperature control system.

3D models of equipment that performs manufacturing process operations were also created in Solid Edge CAD. The main models are shown in Fig. 2. These models serve as the basis for both visualizing equipment in a digital mockup and setting the kinematics of its moving elements, which has a direct impact on the temporal characteristics of assembly and changeover operations necessary to determine the resulting productivity of the assembly area.

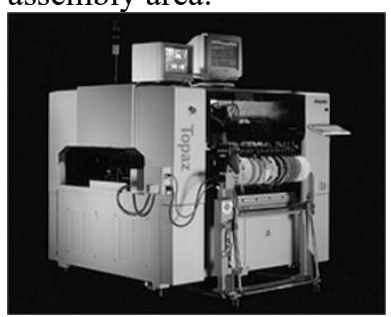

(a)

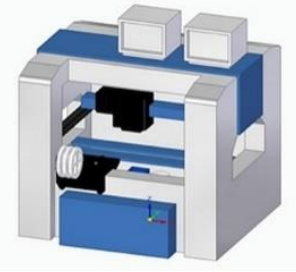

(d)

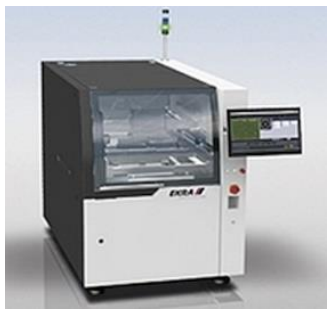

(b)

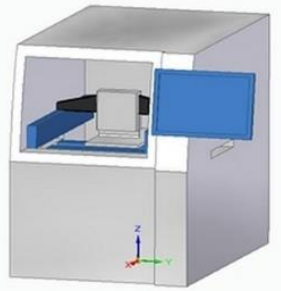

(e)

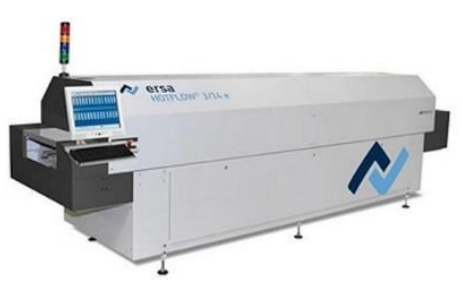

(c)

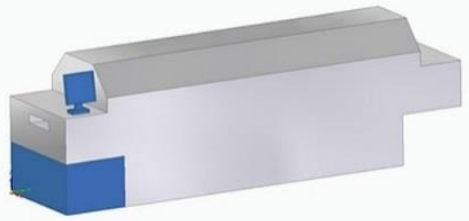

(f)

Fig. 2. General view and 3D CAD models of the main equipment for assembling electronic modules on printed circuit boards: (a), (d) component pick-and-place machine Philips Topaz; (b), (e) solder paste screen printing machine Erka X5 Professional; (c), (f) reflow oven Ersa HotFlow 3 / 14e. 
For example, a conveyor system for transporting a printed circuit board, a block of assembly heads (Fig. 3a) and a block of feeders for electronic components with tape-andreels (Fig. 3b), vision system cameras, monitors for visualizing the progress and parameters of the operation are implemented as a part of the component pick-and-place machine. Subsequently, when setting up the equipment kinematics, attributes will be assigned to these elements.

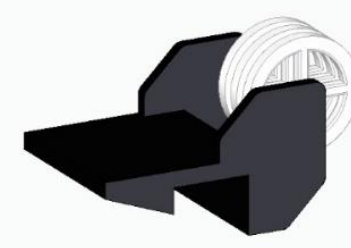

(a)

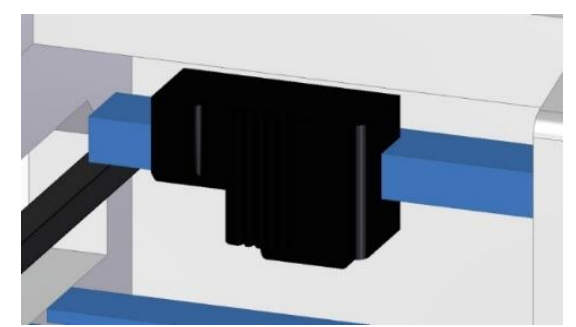

(b)

Fig. 3. Elements of the component pick-and-place machine: (a) block of assembly heads; (b) block of tape feeders.

\subsection{Seeding the Plant Simulation database}

In the Plant Simulation software environment, attributes are assigned to production objects, which are implemented as a hierarchical class library, among which, in particular, moving objects (MUs), Resources and material flow objects (MaterialFlow) can be highlighted.

Moving objects move around the assembly area and are transformed during production. In this project, such an object is an assembly that goes from a bare circuit board to a finished assembly through intermediate states with partially placed / mounted components. All these states, corresponding to certain stages of the assembly process, are described using attributes that include the overall dimensions of the module, parameters, graphics, and the number of components placed / mounted at this stage, and are set in the database. Three such consecutive states of the production object are presented in Fig. 4.

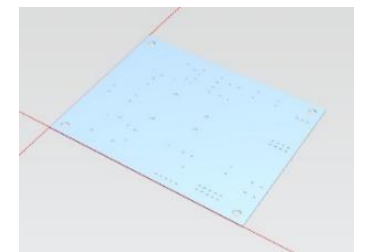

(a)

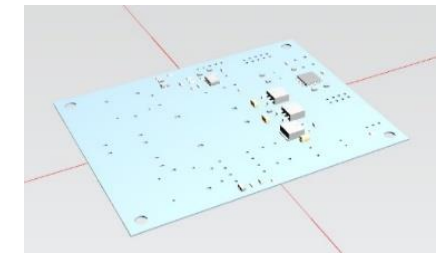

(b)

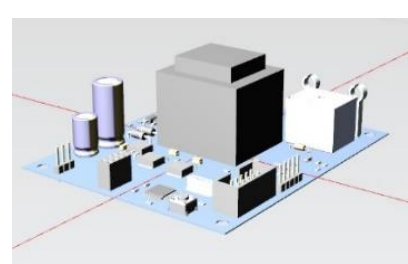

(c)

Fig. 4. The states of the moving production object recorded in the Plant Simulation database: (a) initial state - bare board; (b) one of the intermediate states - board with placed surface mount components, (c) the final state - finished assembly.

Material flow objects are objects that perform some kind of action on moving objects. Among the objects set in the Plant Simulation database, we highlight the main ones:

- Source - a supplier of movable objects with its delivery mode, the number of delivered objects and time intervals between deliveries;

-Drain - a container where, after passing through the production cycle, a moving object gets in, and which collects, stores and allows to display all the information about the moving object, the path and time of its processing;

- Connector - an object linking individual equipment units to each other in order to exchange movable objects; 
- Single machine (SingleProc), Parallel machine (ParallelProc), Assembly station (Assembly) and Disassembly station (DismantleStation) - material flow objects that transform mobile objects, i.e. in this project - manufacturing equipment of various purposes.

- Buffer - an object that accumulates moving objects during the process, i.e. in our case, first of all - the loader and unloader of bare printed circuit boards and finished products, respectively.

- Sorter - an object directing a moving object along one of the given paths depending on the fulfillment of a certain given condition;

-Conveyor line (Line) - an object that allows to create connections between the individual elements of manufacturing equipment and combine them into a single manufacturing line. In addition to the ability of creating links, the line has parameters such as time, the speed of the object and the length of the line itself, and also has the function of animating the movement of the object along it.

To create the geometry of objects, one can use the built-in Plant Simulation tools and create geometry from primitives, such as, for example, a sphere or a box. In addition, it is possible to import models created using third-party CAD systems, for example, Solid Edge. Next, the functionality of the objects and indicators is configured, which helps user to visualize the parameters of the production floor elements (displays, status indicator, etc.).

A single machine is suitable for the implementation of screen printing and component pick and place machines, and to create a simulation model of a reflow oven, on the contrary, a parallel machine should be used, since it can contain several soldered assemblies that simultaneously move along a conveyor line inside the oven. In order for the internal machine to communicate with the external model, one need to add interfaces and connect them to the machine using connectors.

For the model of the material flow object, in addition to the main and auxiliary operations, one can configure failures, each of which is characterized by a certain set of parameters. In this way, various types of failures can be realized, for example, component jamming in the vacuum nozzle of the assembly head, incorrect recognition of the component by vision system cameras, absence of a printed circuit board, etc. These failures can occur at different times and have different solutions. When adding a failure, in particular, the availability of the machine in percent and the average recovery time are set.

To correctly enter the time parameters of the machine, it is necessary to set its kinematics. This is done at the stage of specifying animation for objects when the imported equipment 3D model is decomposed (possibly several times) to provide the ability to select and configure individual functional units of equipment. These elements are converted into an animated object, after which a set of parameters appear that allows to simulate the unit kinematics. Kinematics is adjusted using anchor points with their own sets of coordinates that make up the animation path along which the animated object will move. The angle of unit rotation and the time of its movement between adjacent positions are also set. Each path can contain a large number of points, thus simulating the complex motion kinematics. An example of kinematic setup for the component pick and place machine is shown in Fig. 5 . 


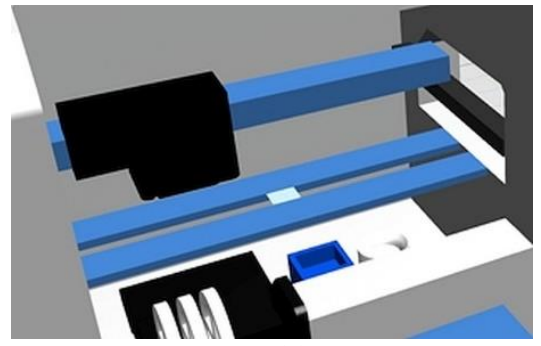

(a)

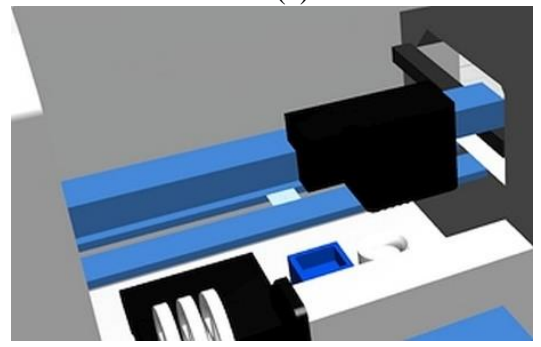

(c)

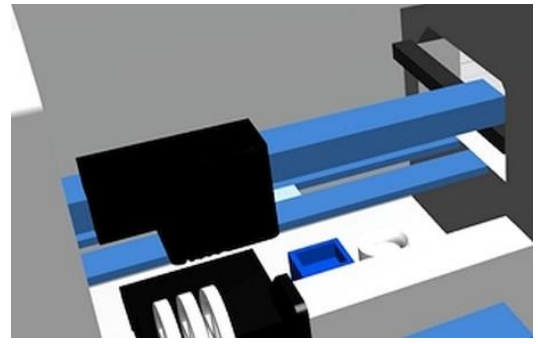

(b)

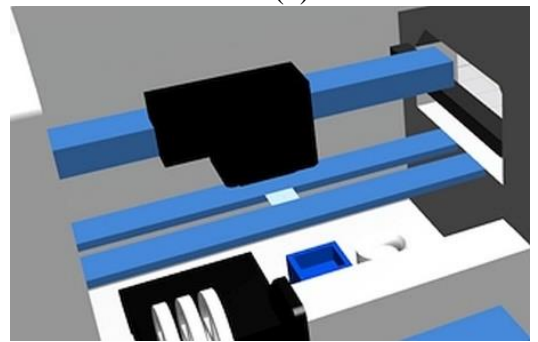

(d)

Fig. 5. The positions of the individual units of the component pick and place machine when setting up its kinematics: a) the initial position of the block of assembly heads; b) the movement of the block of heads along feeders with picking up components; c) carrying picked up components over the on-thefly cameras of the vision system; d) stop at the component placement position.

After creating the animation paths, a method is designed that allows to play all the animations in the required order. The method is created in the second version of the objectoriented programming language SimTalk, developed specifically for the Plant Simulation environment. In order for the designed method to work, it must be linked to an event. Such an event may be the loading of a moving object into the machine, unloading from it, changeover, failure, etc. Thus, the method will be the handler of the corresponding event.

Having completed this procedure with all user material flow objects, we complete the seeding of the Plant Simulation database.

\subsection{Manufacturing line and personnel setup}

Then, on the basis of the selected assembly process, manufacturing equipment, fixtures and tools and a Plant Simulation database seeded by a process engineer, a manufacturing assembly line is created consisting of a source, a line of manufacturing equipment, conveyor lines, drain, as well as personnel.

To simulate the work of personnel, the model provides for special objects from the Resources category, among which there are WorkerPool objects (source of employees) and Broker (employee itself). At that, the source of the worker appearance, the main worker Workplace, as well as the actions performed by him, are configured.

The final customized simulation model of the assembly line is shown in Fig. 6. 


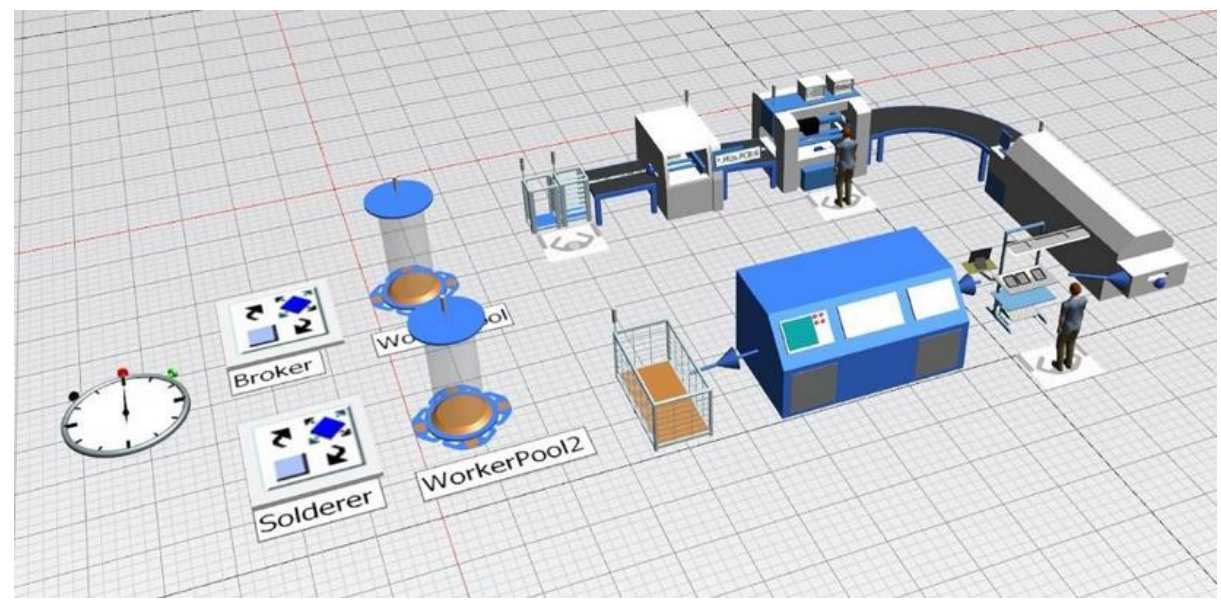

Fig. 6. The final simulation model of the assembly line.

In some cases, there is a need to plan the layout of the production floor and arrange equipment in a limited space. For these purposes, Plant Simulation has a set of ready-made geometric objects, among which there are racks, walls, boxes, barriers, stairs and slabs. These elements allow to set their geometric dimensions, material, color, as well as transparency and lighting parameters. Fig. 7 shows the final view of the created model of the assembly area using walls and floor slab.

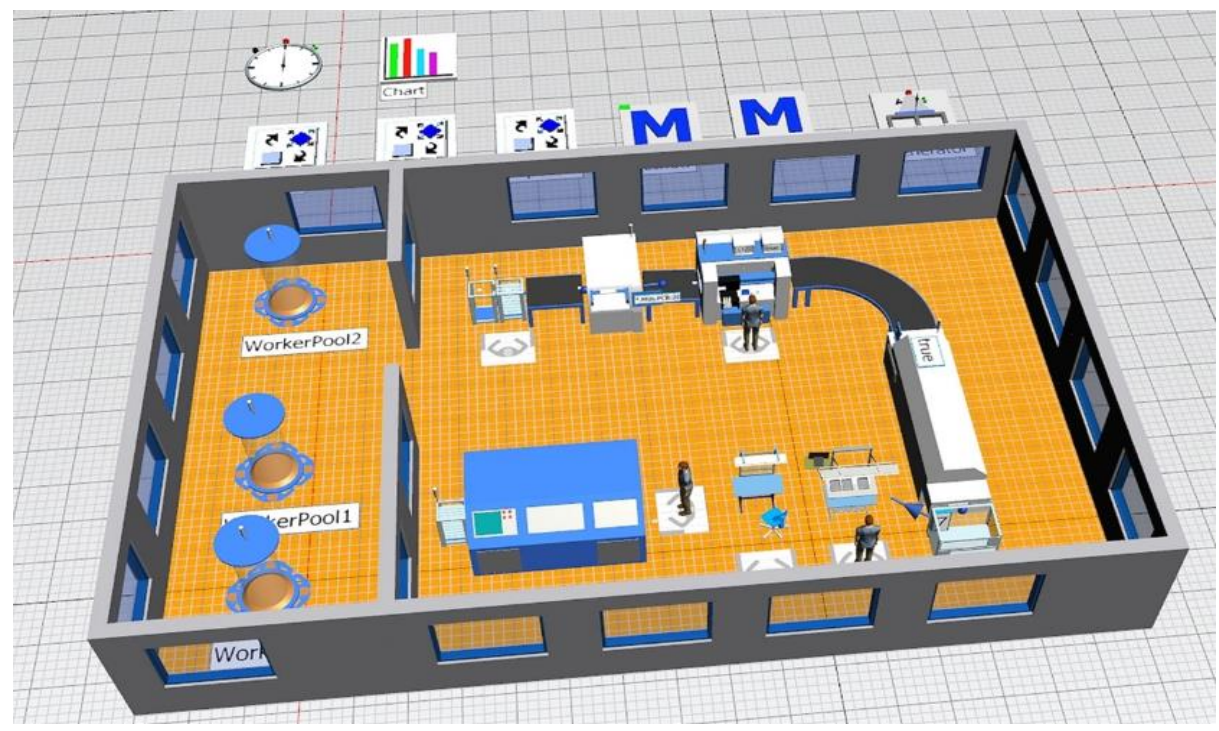

Fig. 7. The final simulation model of the assembly area using walls and floor slabs.

\subsection{Simulation start}

The control of simulation process over time is carried out by an event controller (EventController object). It is installed in the model body and allows to start and pause the simulation, reset the time and adjust the speed of the process. The controller option allows to simulate the process in real time. 


\section{Analysis of the results}

Status visualization and model analysis in Plant Simulation are done in several ways. One of them is the use of the Chart information flow object, which displays statistics on the object use in graphical form. It is also possible to obtain summary statistics for several selected objects in a table form. This table displays statistics on the status of each object, the properties of the material flow, the main time characteristics (waiting, work, changeover, etc.), as well as accumulated statistics of resources, summary statistics of classes and statistics on personnel.

The main results of simulation based on the created model of the assembly line with one mounter workplace are presented in Fig. 8 and Table 1.

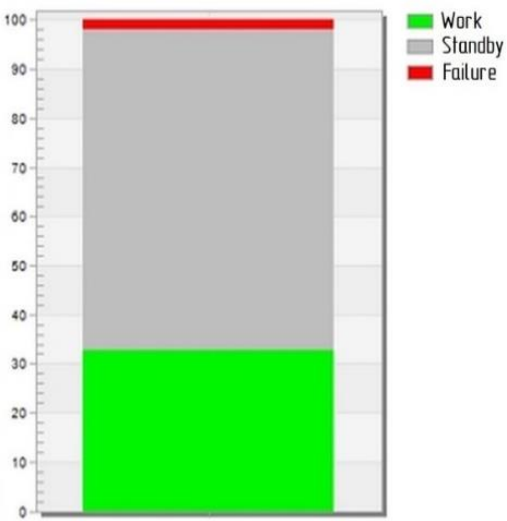

(a)

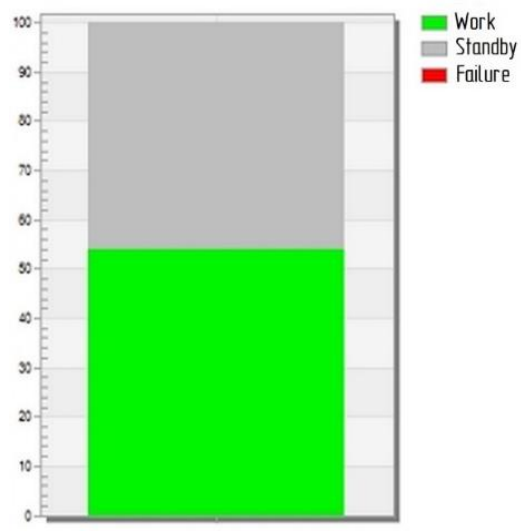

(b)

Fig. 8. The main results of simulation based on the created model of the assembly line with one mounter workplace: (a) statistics of the component pick-and-place machine Philips Topaz; (b) statistics of the reflow oven Ersa HotFlow $3 / 14 \mathrm{e}$.

Table 1. Summary operation statistics of the assembly line with one mounter workplace.

\begin{tabular}{cc}
\hline Parameter & Value \\
\hline Total manufacturing cycle time & $93 \mathrm{~h} 59 \mathrm{~min}$ \\
Assembly performance & 9 modules $/ \mathrm{h}$ \\
Ekra X5 Professional utilization & $2.2 \%$ \\
Philips Topaz utilization & $32.4 \%$ \\
Ersa HotFlow 3/14e utilization & $55.1 \%$ \\
Mounter workplace utilization & $88.2 \%$ \\
Inspector workplace utilization & $18.7 \%$ \\
Cleaning machine utilization & $6.5 \%$ \\
\hline
\end{tabular}

One of the advantages of simulation in comparison with full-scale experiments is the possibility of virtual implementation of various line operation scenarios of the "what if" type. Changes to the digital model can be made at any time, and the results of such a change can be visualized almost immediately.

We give one example of improving the model with working out a new scenario. Analyzing the simulation results shown in Fig. 8 and Table 1, one can note a significant automated equipment underutilization, since with such organization of workplaces, manual soldering takes most of the process time, and automatic pick and place and reflow soldering machines are in standby mode for most of the time. 
To solve this problem, it is necessary to change the number of workplaces to carry out line balancing for productivity. We introduce two additional workers who insert and solder through hole components. The improved model using three mounter workplaces and the corresponding simulation results are shown in Fig. 9 and Table 2.

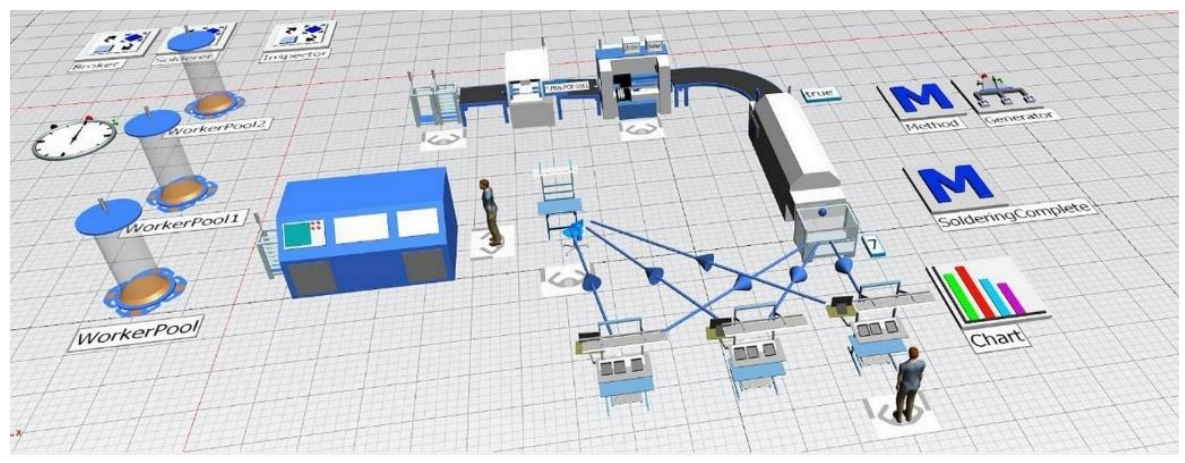

(a)

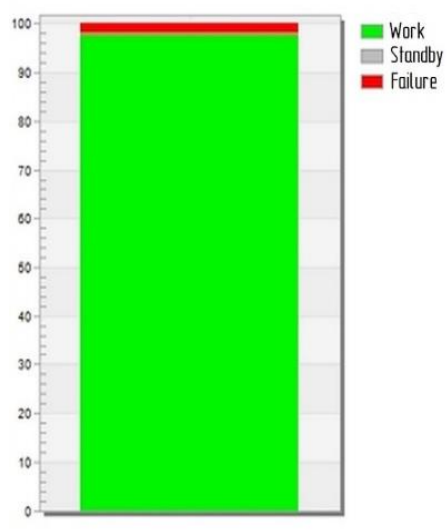

(b)

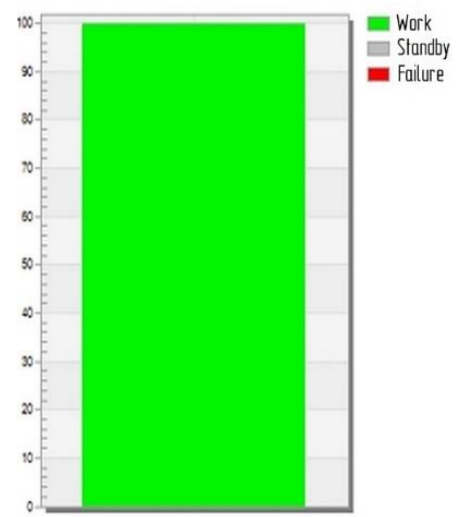

(c)

Fig. 9. The improved simulation model of the assembly line using three workplaces of installers instead of one and the corresponding simulation results: (a) layout of the improved simulation model; (b) statistics of the component pick-and-place machine Philips Topaz; (c) statistics of the reflow oven Ersa HotFlow 3 / 14e.

Table 2. Summary operation statistics of the assembly line with three mounter workplaces.

\begin{tabular}{cc}
\hline Parameter & Value \\
\hline Total manufacturing cycle time & $45 \mathrm{~h} 24 \mathrm{~min}$ \\
Assembly performance & 23 modules $/ \mathrm{h}$ \\
Ekra X5 Professional utilization & $4.1 \%$ \\
Philips Topaz utilization & $95.7 \%$ \\
Ersa HotFlow 3/14e utilization & $100 \%$ \\
Mounter workplace utilization & $97.8 \%$ \\
Inspector workplace utilization & $40.4 \%$ \\
Cleaning machine utilization & $15.1 \%$ \\
\hline
\end{tabular}


The results obtained indicate a close to $100 \%$ utilization of equipment, which leads to 2.5 higher performance indicators of this line working scenario compared to the original version in terms of the number of manufactured products per unit time.

The introduction of these approaches into educational techniques allows to visually familiarize students with discrete production processes, their input and output parameters, study their work after introduction of disturbing influences, perform analysis of results and optimization without the need for presence in physical production floors. Based on the proposed approach, it is possible to develop a generalized methodology for the use of simulation modeling tools in education, which includes stages such as formalizing a problem, creating a model, setting input parameters and scenarios that reveal the essence of the process under study, performing simulation, visualizing and analyzing the results, writing tasks-scenarios for students, discussion of the results and approaches to the improving of the object of study.

The advantage of this approach is a clear interconnection between the digital model and real production, which will contribute to the formation of not abstract, but real skills among students. In addition, learning on a digital model will provide significant savings in resources and costs of the educational process without reducing its quality.

The considered approaches have found wide application in the implementation of modern educational techniques in the context of the economy and manufacturing digitalization. When organizing and conducting technological business games, simulation tools give a high synergistic effect [9-11, 19]. In particular, when training teams for participation in engineering competitions, for example, robotic ones, these approaches, methods and tools allow detailed modeling of the robots' behavior according to a given competition program $[4,5]$.

The proposed simulation technique finds application, in particular, in the development of robotic systems [12], in the design of smart systems for various purposes [16, 20, 21], in educational systems for simulation and research of control and production systems [13-15, $17,18]$.

\section{Conclusion}

Methods and simulation tools used to develop a digital model of the assembly area of electronic modules on printed circuit boards can be widely used in the educational field.

The model created within the framework of the completed project makes it possible to consider many scenarios of composition, layout, staffing, organization of the manufacturing process, etc., which allows to get rid of bottlenecks in terms of assembly productivity, balance manufacturing lines, load manufacturing equipment evenly and at a high level. The resulting model is a digital mockup that allows to visually study the basic tools for creating, analyzing and managing digital production.

The model presented in the paper has the potential in terms of complicating the set kinematics of equipment, increasing flexibility in assembly of various typical products with different set of components, organizing parallel assembly on separate operations, implementing of a full-scale changeover operation with the organization of material and logistics flows between equipment and the storage area for components, fixtures, tools and finished products. These problems are the directions for further work on the model. 


\section{References}

1. A.I. Vlasov, L.V. Juravleva and V.A. Shakhnov, Visual environment of cognitive graphics for end-to-end engineering project-based education, Journal of Applied Engineering Science, vol. 17(1), pp. 99-106 (2019)

2. A.A. Demin and A.I. Vlasov, Visual methods of formalization of knowledge in the conditions of the synchronous technologies of system engineering, ACM International Conference Proceeding Series, 3166098 (2017). DOI: 10.1145/3166094.3166098.

3. V.A. Shakhnov, L.V. Juravleva and A.I. Vlasov, Concept of automated support to problem: modular vocational training Handbook of Research on Engineering Education in a Global Context, IGI Global, pp. 101-114 (2019)

4. A.V. Yudin, A.I. Vlasov, M. Salmina and V. Sukhotskiy, Challenging intensive project-based education: short-term class on mobile robotics with mechatronic elements, Advances in Intelligent Systems and Computing, vol. 829, pp. 79-84 (2019). DOI: 10.1007/978-3-319-97085-1_8.

5. A. Yudin, M. Kolesnikov, A. Vlasov and M. Salmina, Project oriented approach in educational robotics: From robotic competition to practical appliance, Advances in Intelligent Systems and Computing, vol. 457, pp. 83-94 (2017)

6. E. Haller, E. F. Schiller and I. Hartel, Impact of the Digital Factory on the Production Planning Process. Integrating Human Aspects in Production Management, IFIP International Conference for Information Processing, vol. 160, pp. 73-84 (2005)

7. R.K. Martijn Mes, Simulation Modelling using Practical Examples: A Plant Simulation Tutorial, Software version 13.0 (Netherlands: University of Twente), pp. 20-86 (2017)

8. S. Bangsow, Tecnomatix Plant Simulation: Modeling and Programming by Means of Examples (Springer International Publishing Switzerland) (2016)

9. A.A. Aleksandrov, P.A. Kapyrin, N.A. Meshkov, K.A. Neusypin, A. E. Popovich and A. V. Proletarsky, Gamification in the advanced higher professional education: fundamentals of theory and experience of use, International Journal of Civil Engineering and Technology, vol. 9 (11), pp. 1800-1808 (2018)

10. A.E. Kurnosenko and D.I. Arabov, Optimization of electronic components mounting sequence for 3D MID assembly process, Proc. Breakthrough directions of Scientific, Development Perspectives in the Framework of the Strategic. KnE Engineering, vol. 3 (6), pp. 311-321 (2018). DOI: 10.18502/keg.v3i6.3009.

11. K.A. Neusypin, M.S. Selezneva and T.Yu. Tsibizova, Diagnostics algorithms for flight vehicles navigation complex, International Russian Automation Conference (RusAutoCon) electronic edition, pp. 1-6 (2018)

12. A. Yudin, A. Vlasov, M. Shalashova and M. Salmina, Evolution of educational robotics in supplementary education of children, Intelligent Systems and Computing, vol, 1023, pp. 336-343 (2020)

13. L. Toomsalu, S. Tolmacheva, A. Vlasov and V. Chernova, Determinants of innovations in small and medium enterprises: a european and international experience, Terra Economicus, vol. 17, no. 2, pp. 112-123 (2019)

14. G.N. Todorov, E.E. Volkova, A.I. Vlasov and N.I. Nikitina, Modeling energy-efficient consumption at industrial enterprises, International Journal of Energy Economics and Policy, vol. 9, no. 2, pp. 10-18 (2019)

15. V.A. Shakhnov, S.S. Filin, A.I. Vlasov and A.I. Krivoshein, Sustainable energy systems in the digital economy: concept of smart machines, Entrepreneurship and Sustainability Issues, vol. 6, no. 4, pp. 1975-1986 (2019) 
16. O.N. Berdyugina, A.I. Vlasov and A.I. Krivoshein, Technological platform for innovative social infrastructure development on basis of smart machines and principles of internet of things, Proceedings Global Smart Industry Conference (GloSIC), 8570062 (2018)

17. I.N. Popova. A. Vlasov and N.I. Nikitina, Optimization of inventory distribution logistics in industrial enterprises, Espacios, vol. 39, no. 24 (2018)

18. V. Akberdina, A. Kalinina and A. Vlasov, Transformation stages of the Russian industrial complex in the context of economy digitization, Problems and Perspectives in Management, vol. 16, no. 4, pp. 201-211 (2018)

19. K.A. Neusypin, M.S. Selezneva, A.V. Proletarsky and K. Shen, Algorithm for building models of ins/gnss integrated navigation system using the degree of identifiability, 25th Saint Petersburg International Conference on Integrated Navigation Systems, ICINS 2018 Proceedings, pp. 1-5 (2018)

20. B.G. Ilyasov, E.S. Zakieva and E.I. Filosova, Simulation modeling system of the life quality dynamics, Information Innovative Technologies, no. 3, pp. 534-543 (2014)

21. Yu.I. Brodsky, Model-oriented programming - CAD methods in simulation modelling, Information Innovative Technologies, no. 1, pp. 142-146 (2017) 